\title{
Radiation-Induced Imaging Changes and Cerebral Edema following Stereotactic Radiosurgery for Brain AVMs
}

(D)B.J. Daou, (D) G. Palmateer, (DD.A. Wilkinson, (DB.G. Thompson, (D).O. Maher, (D) N. Chaudhary, (D).J. Gemmete, (D).A. Hayman, (D) K. Lam, DD.R. Wahl, (D) Kim, and (D)A.S. Pandey

\begin{abstract}
BACKGROUND AND PURPOSE: T2 signal and FLAIR changes in patients undergoing stereotactic radiosurgery for brain AVMs may occur posttreatment and could result in adverse radiation effects. We aimed to evaluate outcomes in patients with these imaging changes, the frequency and degree of this response, and factors associated with it.
\end{abstract}

MATERIALS AND METHODS: Through this retrospective cohort study, consecutive patients treated with stereotactic radiosurgery for brain AVMs who had at least 1 year of follow-up MR imaging were identified. Logistic regression analysis was used to evaluate predictors of outcomes.

RESULTS: One-hundred-sixty AVMs were treated in 148 patients (mean, 35.6 years of age), including 42 (26.2\%) pediatric AVMs. The mean MR imaging follow-up was 56.5 months. The median Spetzler-Martin grade was III. The mean maximal AVM diameter was $2.8 \mathrm{~cm}$, and the mean AVM target volume was $7.4 \mathrm{~mL}$. The median radiation dose was $16.5 \mathrm{~Gy}$. New T2 signal and FLAIR hyperintensity were noted in $40 \%$ of AVMs. T2 FLAIR volumes at 3, 6, 12, 18, and 24 months were, respectively, 4.04, 55.47, 56.42, 48.06, and $29.38 \mathrm{~mL}$ Radiation-induced neurologic symptoms were encountered in $34.4 \%$. In patients with radiation-induced imaging changes, $69.2 \%$ had new neurologic symptoms versus $9.5 \%$ of patients with no imaging changes $(P=.0001)$. Imaging changes were significantly associated with new neurologic findings $(P<.001)$. Larger AVM maximal diameter $(P=.04)$ and the presence of multiple feeding arteries $(P=.01)$ were associated with radiation-induced imaging changes.

CONCLUSIONS: Radiation-induced imaging changes are common following linear particle accelerator-based stereotactic radiosurgery for brain AVMs, appear to peak at 12 months, and are significantly associated with new neurologic findings.

ABBREVIATIONS: ARE = adverse radiation effects; LINAC = linear particle accelerator; SRS = stereotactic radiosurgery

S tereotactic radiosurgery (SRS) has become a standard tool for treating brain arteriovenous malformations (AVMs), especially for AVMs in deep or eloquent locations and those with complex angioarchitecture for which surgical treatment and endovascular embolization are more challenging and riskier. AVM obliteration rates of about $80 \%$ have been reported with long-term follow-up. ${ }^{1-3}$ Radiosurgery, however, results in gradual obliteration of the AVM over several years following treatment. During this time period and until the AVM is obliterated, patients continue to be at risk of intracranial hemorrhage. ${ }^{4}$ Patients are routinely followed with brain MRI/MRA during this latency period, with close monitoring for

Received April 30, 2020; accepted after revision August 17.

From the Departments of Radiation Oncology (J.A.H., K.L., D.R.W., M.K.), Neurosurgery (B.J.D., G.P., D.A.W., B.G.T., C.O.M., N.C., J.J.G., A.S.P.), Radiology (N.C., J.J.G.), and Radiation Oncology (J.A.H., K.L., D.R.W., M.K.), University of Michigan, Ann Arbor, Michigan.

Please address correspondence to Aditya S. Pandey, MD, Department of Neurosurgery, University of Michigan, 1500 E Medical Center Dr, 3552 Taubman Center, Ann Arbor, Ml 48109-5338; e-mail: adityap@med.umich.edu

http://dx.doi.org/10.3174/ajnr.A6880 treatment-related adverse effects, including cerebral edema and radiation-induced necrosis. Following SRS, the hemodynamics of the treated AVM are progressively altered, and various degrees of T2 signal and Fluid-attenuated inversion recovery (FLAIR) hyperintensity, suggestive of cerebral edema, have been observed in these patients. ${ }^{1,5,6}$ In addition, notable adverse radiation effects (ARE) with new neurologic signs and symptoms may arise, particularly during this time period and may affect patient outcomes. ${ }^{6}$

In this study, we aimed to investigate the characteristics of the T2 signal and FLAIR response on follow-up MRIs in patients undergoing SRS for brain AVMs, evaluate the frequency of these radiation-induced imaging changes, attempt to quantify the degree of T2 signal and FLAIR changes, identify factors associated with this response, and evaluate its impact on patient outcomes.

\section{MATERIALS AND METHODS Patient Selection}

The study protocol was approved by the institutional review board. The informed consent of patients was not required for this 
retrospective review of medical records. A combination of International Classification of Diseases codes for brain AVMs in association with the Current Procedural Terminology code for SRS was used to identify our target population. Electronic medical records were retrospectively reviewed to identify patients with the diagnosis of cerebral AVM treated with SRS during a 28-year period. Our institutional Electronic Medical Record Search Engine data base was used for patient identification and data collection. $^{7}$

All patients with brain AVMs confirmed with diagnostic cerebral angiography and treated with SRS between January 1990 and December 2018 were included in the initial analysis, resulting in 210 patients with 222 treated AVMs. Exclusion criteria were patients who lacked at least 1 year of clinical follow-up as well as MR imaging follow-up ( $n=62$ patients). Patients who were followed clinically but without available brain MRIs that included T2 and T2 FLAIR sequences for analysis were excluded. Post-SRS hemorrhage was encountered after treatment of 10 AVMs in the study population. The hemorrhage was not fatal in 8 patients who continued to get follow-up and was fatal in 1 patient and occurred at 18 months post-SRS. Therefore, these patients were included in the study population. One additional fatal post-SRS hemorrhage was excluded, given that the event occurred at 6 months (did not meet inclusion criteria).

\section{Variables and Outcomes}

We evaluated patient age, sex, clinical presentation, treatment time period, AVM maximal diameter, venous drainage, eloquence, previous embolization, the Spetzler-Martin grading scale, AVM location, angioarchitecture of the AVM (including the presence of multiple arterial feeders or multiple draining veins), presence of a venous varix, anatomy of the draining vein (large cortical vein or other), presence of an intranidal aneurysm, delivered radiation dose, nidus volume, and isodose volume, along with clinical, angiographic, and MR imaging followup periods.

The primary end points of this study were the following: 1) to evaluate the frequency of radiation-induced imaging changes, 2) quantify the degree of T2 signal and FLAIR changes, 3) identify factors associated with the development of prominent T2 signal and FLAIR changes, and 4) evaluate the impact of these radiologic changes on patient outcomes.

T2 signal and FLAIR hyperintensity were evaluated on brain MR imaging (patients who did not have a brain MR at least at 1 year posttreatment were not included in the analysis). Radiationinduced imaging changes were defined as new areas of increased T2 and FLAIR signal surrounding the treated AVM on follow-up MR imaging. Volumes were calculated using T2 FLAIR axial sequences, through a section-by-section analysis, and the $\mathrm{ABC} / 2$ technique to quantify the volume of perinidal T2 FLAIR changes. ${ }^{8}$ For each patient, we evaluated all available posttreatment MRIs in a longitudinal fashion. AVM obliteration was determined on diagnostic cerebral angiography and MRI/MRA studies in patients with at least 2 years of imaging follow-up after treatment. All reported new symptoms determined to be related to SRS by the treating team were included under radiation-induced neurologic signs and symptoms.

\section{Procedure Description}

Informed consent was obtained for linear particle accelerator (LINAC)-based stereotactic radiosurgery, and options of conservative management and surgical resection were discussed with the patient. Patients were premedicated with an oral narcotic and anxiolytic before frame placement with administration of a local anesthetic at the pin sites. Patients then underwent placement of a Cosman-Roberts-Wells (CRW) frame. With the CRW frame in place, a CT of the head was performed. These images were fused to a previously obtained contrasted thin-section MR imaging of the brain, and the AVM volume was drawn around the nidus. Depending on the location of the AVM relative to radioeloquent structures (brain stem, optic apparatus, and so forth), the radiation oncology and neurosurgery teams developed a plan to allow maximal dose delivery to the nidus while minimizing the dose to radioeloquent regions. A single dose of dexamethasone, $10 \mathrm{mg}$, was given 1-2 hours before treatment without a taper. Our goal was to deliver $18-20$ Gy to the nidus using a LINAC-based system; however, generally, this dose was reduced in cases in which the AVM was located near a radiosensitive structure. In addition, the dose was also commonly reduced for larger volume AVMs. Post-SRS, patients were discharged and were then imaged with brain MRIs with gadolinium at 6- to 12-month intervals.

\section{Statistical Analysis}

Data are presented as mean and range for continuous variables and as frequency for categoric variables. Analysis was performed using the unpaired $t$ test and $\chi^{2}$ and Fisher exact tests as appropriate. Statistical analysis was performed using STATA (StataCorp). Univariate analysis was used to test covariates predictive of the following dependent outcomes: radiation-induced imaging changes and new neurologic signs or symptoms post-SRS. Factors predictive in univariate analysis $(P<.10)$ were entered into a backward multivariate logistic regression analysis. $P$ values $\leq .05$ were considered statistically significant.

\section{RESULTS}

\section{Baseline Patient Characteristics}

One-hundred-sixty AVMs in 148 patients treated with SRS made up the study population. The mean patient age was 35.6 years (range, 3-75 years). Patients 18 years of age or younger had 42 treated AVMs (26.2\%). There were 84 AVMs (52.5\%) in males and 76 AVMs (47.5\%) in females. Eighty-eight AVMs (55\%) had ruptured and bled before SRS treatment. Twenty-two AVMs (13.7\%) were previously embolized before SRS. The mean clinical follow-up was 61.2 months, the mean MR imaging follow-up was 56.5 months, and the mean angiographic follow-up was 54 months.

\section{AVM and Treatment Characteristics}

The locations of the AVMs were as follows: the frontal lobe in 47 patients (29.4\%), parietal lobe in 24 patients (15\%), temporal lobe in 24 patients (15\%), occipital lobe in 21 patients $(13.1 \%)$, cerebellar in 25 patients $(15.6 \%)$, brain stem in 6 patients $(3.8 \%)$, corpus callosum in 5 patients $(3.1 \%)$, thalamic in 4 patients $(2.5 \%)$, and basal ganglia in 4 patients $(2.5 \%)$. The median SpetzlerMartin grade was 3. Thirteen AVMs (8.1\%) were classified as 
Spetzler-Martin grade I; $42(26.3 \%)$, as grade II; $71(44.4 \%)$, as grade III; 29 (18.1\%), as grade IV; and $5(3.1 \%)$, as grade V. The mean maximal AVM diameter was $2.8 \mathrm{~cm}$ (range, $0.5-6.3 \mathrm{~cm}$ ). One hundred thirty-four AVMs (83.8\%) were determined to be in an eloquent location. Seventy-one treated AVMs (44.4\%) had deep venous drainage. Seventy AVMs (43.8\%) had multiple draining veins, and 95 (59.4\%) had multiple feeding arteries. A venous varix was present in 13 AVMs (8.1\%), and an associated perinidal or intranidal aneurysm, in 23 AVMs (14.4\%).

The mean AVM target volume was $7.4 \mathrm{~mL}$, the mean isodose surface volume was $12.5 \mathrm{~mL}$, and the median radiation dose was $16.5 \mathrm{~Gy}$.

\section{Radiation-Induced Imaging Changes}

New T2 signal and FLAIR hyperintensity were noted following treatment of 64 AVMs (40\%) (Fig 1). We divided the MR imaging observations into 6 time periods following SRS. At 3 months, 13 MRIs were available for analysis, and 7 (53.8\%) were positive for imaging changes, with a mean T2 FLAIR volume of $4.04 \mathrm{~mL}$. Thirty-nine patients had MRIs at 6 months, and 28 (71.8\%) had

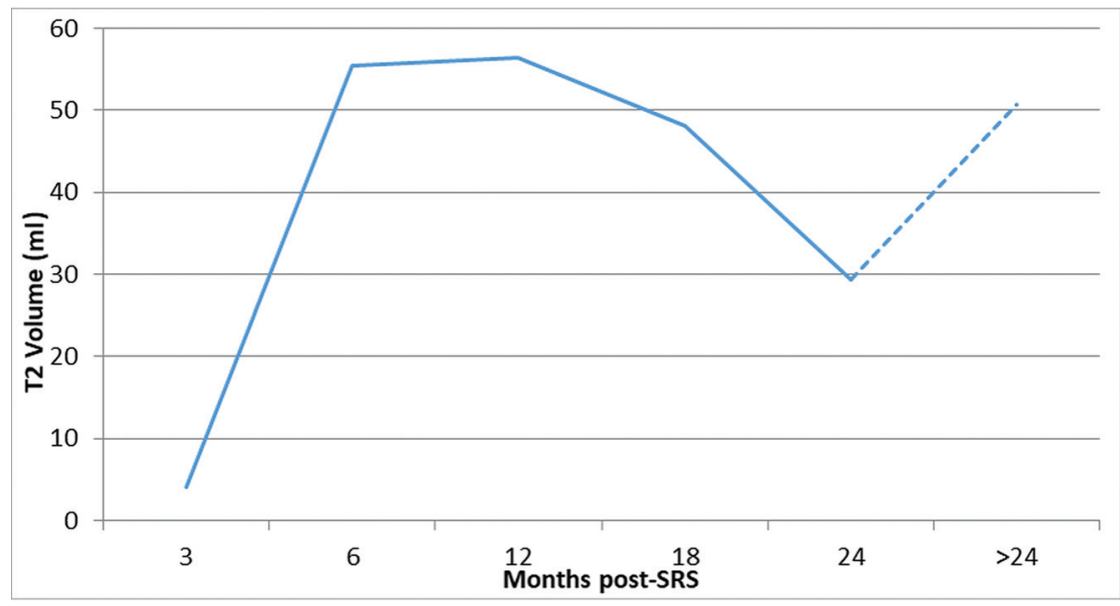

FIG 1. Evolution of radiation-induced imaging changes, with evaluation at $3,6,12,18,24$, and $>24$ months. imaging changes with a mean T2 FLAIR volume of $55.47 \mathrm{~mL} ; 131$ patients had MRIs at 1 year, with 52 patients (39.7\%) having radiation changes with a mean T2 FLAIR volume of $56.42 \mathrm{~mL} ; 54$ patients had MRIs at 18 months with 25 patients (46.3\%) having T2 FLAIR changes with a volume of $48.06 \mathrm{~mL}$; and 93 patients had MRIs at 2 years, 34 of whom (36.6\%) had radiation-induced changes with a mean T2 FLAIR volume of $29.38 \mathrm{~mL}$. In addition, in 78 patients with MRIs at $>2$ years, 29 patients $(37.2 \%)$ had radiation-induced imaging changes with a mean T2 FLAIR volume of $50.76 \mathrm{~mL}$, reflecting some cases that developed delayed prominent T2 FLAIR signal change. Specifically, new T2 FLAIR signal changes were delayed or worsened $>2$ years after SRS in 8 cases $(5 \%$ of the total cases and $12.5 \%$ of the cases developing prominent T2 FLAIR signal change posttreatment) (Fig 2).

\section{Radiation-Induced Neurologic Signs and Symptoms}

New radiation-induced neurologic signs and symptoms were encountered in 55 cases (34.4\%). These included headaches following 30 treatments $(18.7 \%)$, new seizures following 16 treatments $(10 \%)$, new cognitive deficits in 6 cases $(3.7 \%)$, a new focal deficit (including motor or cranial nerve deficit) in 10 cases (6.2\%), severe nausea/vomiting after 8 treatments (5\%), unsteadiness/imbalance in 7 cases $(4.4 \%)$, transient sensory symptoms in 6 cases $(3.7 \%)$, and word-finding difficulties in 2 patients (1.2\%).

Three patients developed a cyst in the radiated region, and 2 patients developed a cavernoma in the SRS region on follow-up. Cysts were diagnosed at 29, 54, and 62 months postSRS, and cavernomas, at 9 and 11 years post-SRS. All 5 patients underwent surgical treatment of these acquired lesions, which were symptomatic in all cases. Four of these 5 patients had

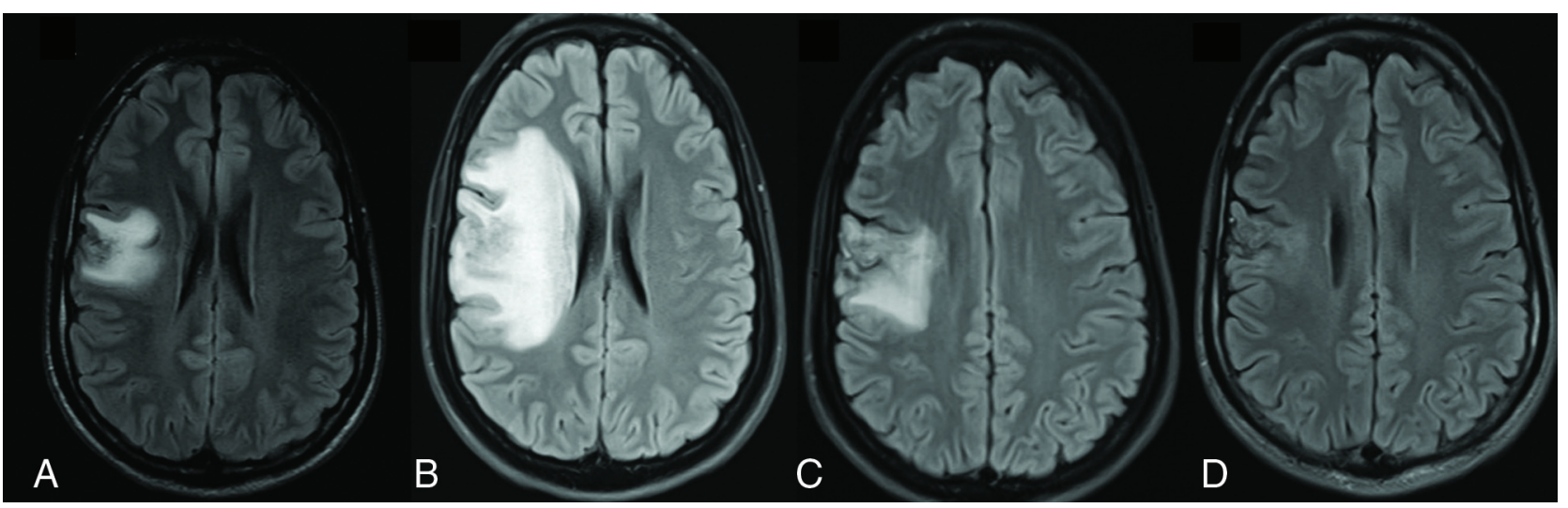

FIG 2. Case illustration of a 17-year-old adolescent boy with a frontal arteriovenous malformation. He developed new seizures 6 months following stereotactic radiosurgery. MR imaging $(A)$ shows an area of increased FLAIR signal change. The FLAIR changes increased and peaked at 12 months $(B)$. At 18 months $(C)$, there were persistent but reduced FLAIR changes, and at 24 months $(D)$, these imaging changes had resolved. 
developed prominent T2 and FLAIR signal changes early after treatment.

\section{Association of Radiation-Induced Imaging Changes with Outcomes}

In patients who developed radiation-induced imaging changes, 45/ 65 patients $(69.2 \%)$ had new neurologic signs or symptoms postSRS. In patients who did not develop imaging changes, 9/95 patients $(9.5 \%)$ had new neurologic signs or symptoms $(P=.0001)$.

One hundred twenty-two patients had 2-year imaging followup to assess AVM obliteration. In patients who developed radiation-induced imaging changes, 39/46 patients (84.8\%) had complete obliteration of their AVMs and 7/46 patients (15.2\%) had incomplete AVM obliteration. In patients who did not develop imaging changes, 55/76 patients (72.4\%) had complete AVM obliteration and 21/76 (27.6\%) had incomplete AVM obliteration. This difference approached significance with $P=.1$.

\section{Factors Associated with Radiation-Induced Imaging Changes}

In univariate and multivariate analyses, Spetzler-Martin grade IV $(\mathrm{OR}=7.03$; 95\% CI, 1.5-32; $P=.012)$, larger maximal diameter (OR $=1.3 ; 95 \% \mathrm{CI}, 1.01-1.67 ; P=.04)$, and the presence of multiple feeding arteries $(\mathrm{OR}=2.4 ; 95 \% \mathrm{CI}, 1.2-4.7 ; P=.01)$ were associated with radiation-induced imaging changes.

The occurrence of new neurologic signs and symptoms postSRS was significantly associated with the presence of radiationinduced imaging changes in both univariate and multivariate analysis $(\mathrm{OR}=20$; 95\% CI, 8.4-47.6; $P<.001)$.

\section{Factors Associated with New Neurologic Signs and Symptoms Post-SRS}

In univariate analysis, AVMs with multiple feeding arteries $(\mathrm{OR}=$ 2.4; 95\% CI, 1.2-4.9; $P=.01$ and the presence of radiation-induced imaging changes after SRS $(\mathrm{OR}=20 ; 95 \% \mathrm{CI}, 8.4-47.6 ; P<.001)$ were associated with new neurologic signs and symptoms post-SRS. In multivariate analysis, only the development of radiation-induced imaging changes was associated with $\mathrm{ARE}(\mathrm{OR}=19.3$; 95\% CI, 7.8-47.8; $P<.001)$.

\section{DISCUSSION}

\section{Rate of Radiation-Induced Imaging Changes}

We reviewed our experience with LINAC-based SRS treatment of brain AVMs and report a high rate of radiation-induced imaging changes in the posttreatment period. Forty percent of patients had prominent T2 signal or FLAIR hyperintensity on follow-up MRIs. The reported frequency of these radiologic changes in the literature has been variable, ranging from $16 \%$ to $62 \%{ }^{5}$ This result is likely related to differences in the interpretation and classification of these findings and variable follow-up times. Lunsford et $\mathrm{al}^{1}$ reviewed follow-up MRIs for 227 patients and reported increased T2 signal in $24 \%$ of patients at a mean interval of 10 months after SRS. In a study that evaluated 107 patients with brain AVMs, in addition to some tumors, Ganz et $\mathrm{al}^{9}$ reported a $60 \%$ incidence of radiationinduced imaging changes. Yen et $\mathrm{al}^{5}$ evaluated 1426 gamma knife surgery procedures and noted radiation-induced imaging changes in $33.8 \%$ of treatments. New T2 signal and FLAIR hyperintensity following SRS are common and are suggestive of a high rate of cerebral edema or radiation necrosis following treatment. Our usual practice involves obtaining MR imaging at 6- to 12-month intervals to evaluate parenchymal and AVM changes posttreatment. The higher rate of radiation-induced imaging changes at 3 and 6 months posttreatment is likely related to patients presenting with symptoms that prompted additional early imaging, therefore resulting in this higher observed rate of imaging changes at 3 and 6 months.

\section{Symptoms Related to Radiation-Induced Imaging Changes}

Despite the high rate of imaging changes, the rate of symptomatic ARE is reported to be much lower, ranging from $3.7 \%$ to $10.8 \%$, with only a very small number of patients having permanent deficits. ${ }^{5}$ Radiation-induced imaging changes were commonly found with new neurologic signs or symptoms following SRS in the present study. This finding is in contrast to that of Lunsford et al, ${ }^{1}$ reporting that $6 \%$ of patients who developed radiologic changes were symptomatic and only $1 \%$ had permanent treatment-related deficits.

In a meta-analysis by Ilyas et $\mathrm{al}^{10}$ on radiation-induced changes following SRS for brain AVMs, the rates of radiologic, symptomatic, and permanent changes were noted to be $35.5 \%$ (1143/3222 patients), 9.2\% (499/5447 patients), and 3.8\% (202/ 5272 patients), respectively. They concluded that approximately 1 in 4 patients who develop radiologic changes will become symptomatic. Yen et $\mathrm{al}^{5}$ reported that $8.6 \%$ of patients with imaging changes developed neurologic symptoms and $1.8 \%$ had permanent deficits. Our analysis shows that the presence of radiationinduced imaging changes is associated with a higher rate of neurologic symptoms than previously reported (69.2\% versus $9.5 \%$ in patients without imaging changes, $P=.0001)$. We agree that these changes result in temporary symptoms in most patients, and permanent symptoms are uncommon. Furthermore, the volume of the new T2 signal change posttreatment varied greatly, very likely affecting the development and severity of symptoms. The developing response on MR imaging reflects underlying edema or radiation necrosis that could result in a significant mass effect, midline shift, and sometimes hydrocephalus.

\section{Proposed Mechanisms Leading to Radiation-Induced Imaging Changes}

The mechanisms behind these T2 and FLAIR changes are not completely understood but are thought to be related to either the direct effects of the radiation treatment on the treated tissue or a vascular/hemodynamic phenomenon resulting in edema or potentially radiation necrosis. ${ }^{5,11,12}$ Mechanisms in which radiation results in these findings through direct tissue damage include injury of glial cells, endothelial cell damage followed by breakdown of the blood-brain barrier, excessive generation of free radicals, and the induction of an autoimmune response. ${ }^{5,13,14}$ Vascular mechanisms inducing imaging changes were first suggested by Pollock ${ }^{15}$ and Chapman et al, ${ }^{16}$ who proposed the concept of occlusive hyperemia, in which local hemodynamic changes can occur if venous outflow is obstructed, with resultant stasis of the blood flow and clot formation in the draining veins. 


\section{Factors Associated with Radiation-Induced Imaging Changes}

Kano et $\mathrm{al}^{11}$ analyzed 755 patients undergoing SRS therapy, examining risk factors for adverse events after radiosurgery, and found that the risk of symptomatic ARE increased with larger AVM volume, higher margin dose, a higher Spetzler-Martin grade, and a higher radiosurgery-based score. They also noted that patients with AVMs located in the brain stem (22\%)/thalamus (16\%) versus other locations (4\%-8\%) were more likely to develop symptomatic ARE. ${ }^{11}$ Cohen-Inbar et $\mathrm{al}^{6}$ examined radiographic changes following gamma knife surgery for AVMs and reported that the radiographic presence of ARE (specifically the ratio of T2 change to nidus volume, or the ARE index) was predicted by nidus volume, deep location, and multiple draining veins. Yen et $\mathrm{al}^{5}$ reported a negative history of prior surgery or prior hemorrhage, large nidus, and a single draining vein to be associated with a higher risk of radiation-induced imaging changes. A study from van den Berg et $\mathrm{al}^{17}$ also reported that nidi with single draining veins were more common in the group of patients with extensive imaging changes than in the those with mild or no imaging changes. Overall, several factors have been reported to be associated with radiation-induced imaging changes, including patient age, AVM size and volume, high-grade AVMs, SRS dose, prior embolization, prior hemorrhage, number of draining veins, and AVM location. ${ }^{5,6,10,11}$

In our analysis, patients with larger AVMs and AVMs with multiple feeding arteries had higher odds of radiation-induced imaging changes. Even though the edema response is thought to be primarily of venous origin, we argue that occlusion of several arterial branches could worsen this phenomenon by increasing the associated arterial injury/thrombosis and worsening the associated ischemic insult with resultant edema adjacent to the nidus.

\section{Radiation-Induced Imaging Changes and AVM Obliteration}

Not only do radiation-induced imaging changes predict ARE, but they are further thought to represent a precursor to AVM obliteration, given similar underlying pathophysiologic mechanisms dictating both processes. In our study, AVM obliteration was achieved in $84.8 \%$ of nidi with surrounding edema versus $72.4 \%$ in patients without this response, with the association approaching significance $(P=.1)$. Yen et $\mathrm{al}^{5}$ reported that $62.8 \%$ of AVMs that developed radiation-induced imaging changes were completely obliterated versus $52.1 \%$ of AVMs without imaging changes $(P<.001)$. They also added that treatment that resulted in extensive imaging findings was more likely to result in AVM obliteration compared with treatment with mild changes. A similar observation was reported by van den Berg et al. ${ }^{17}$ They reported an AVM obliteration rate of $88 \%$ in patients with extensive radiationinduced imaging changes compared with $50 \%$ in patients without extensive signal changes on MR imaging following SRS. The edema response could be related to the AVM obliteration process in which there is premature thrombosis of a draining vein or early occlusion of a feeding artery.

\section{Timing of Radiation-Induced Imaging Changes}

Radiation-induced imaging changes appear to peak at 12 months after treatment. This response continues to be present in most patients who develop these imaging changes at 18 months. However, the response is not uniform and could appear a few months after treatment or $>1-2$ years after SRS. Yen et $\mathrm{al}^{5}$ reported a median duration of 13 months from SRS to the development of radiation-induced imaging changes, with a range of 2124 months. The imaging changes disappeared completely within a median duration of 22 months from their identification. Another study reported that the development of radiationinduced imaging changes follows a temporal pattern, peaking at 7-12 months after SRS. ${ }^{6}$ They also added that if these imaging findings peak at 7-12 months post-SRS, AVMs have higher odds of complete obliteration.

We did observe another late peak in T2 signal and FLAIR hyperintensity $>2$ years from treatment in some patients. CohenInbar et $\mathrm{al}^{6}$ also observed late ARE (appearing at $>1$ year of follow-up) and attributed those late changes to the direct tissue effects of radiation and to an inflammatory process as opposed to a vascular process that could be related to earlier imaging changes. In addition, radiation-induced imaging changes have also been associated with late complications after SRS, such as cyst formation and late-onset edema through a continued inflammatory process. ${ }^{10,18}$ In our study, 3 patients developed a cyst and 2 patients developed a cavernoma, all requiring surgical treatment. Patients who develop radiation-induced imaging changes may require longer clinical and radiologic follow-up.

\section{Management of Symptomatic Radiation-Induced Imaging Changes}

Patients with radiation-induced imaging changes who were symptomatic were treated with an oral regimen of steroids, typically dexamethasone. Patients with severe radiation-induced imaging changes and acute neurologic decline were admitted to the hospital with initiation of intravenous steroids and close neurologic monitoring. Some patients who develop large volumes of T2 or FLAIR signal change could be dependent on steroids for several months, with the potential for worsening if steroids are weaned too quickly. Asymptomatic patients or patients with minimal symptoms should still be considered for medical management if they have extensive imaging changes. The course of steroids was variable and related to the symptoms and imaging findings. Other agents reported to attenuate the radiation-induced imaging changes include pentoxifylline and bevacizumab. ${ }^{19,20}$ Furthermore, extensive imaging changes could result in hydrocephalus, elevated intracranial pressure, and mass effect, which could necessitate surgical measures related to CSF diversion (shunt versus ventriculostomy). None of the patients in our series required a direct surgical intervention to target the imaging changes or their acute consequences. Neurologic follow-up with frequent MR imaging should be pursued in patients with extensive imaging changes.

\section{Limitations}

This study is limited by its retrospective design, the chart-review process, and the inherent selection bias. This study is a singleinstitution series using LINAC SRS and is based on institutional practice, which could affect the generalizability of the study. Furthermore, follow-up brain MRIs were not available for all patients at all time periods, limiting the analysis and impeding us 
from performing a full temporal analysis of the radiation-induced imaging findings. We did not routinely obtain MRIs to document resolution of the imaging changes, and MRIs were obtained as clinically indicated outside the 2-year follow-up MRIs. We documented resolution of the imaging changes in 13/64 patients (20.3\%), though it is likely that more patients had resolution of these imaging findings but were not captured by our study.

\section{CONCLUSIONS}

Radiation-induced imaging changes are common in the posttreatment period following LINAC-based SRS for brain AVMs. These imaging changes are significantly associated with new neurologic signs or symptoms following treatment. Patients with larger AVMs and AVMs with multiple feeding arteries may have higher odds of developing radiation-induced imaging changes. These changes are variable in severity and timing but appear to peak at 12 months.

Disclosures: Daniel R. Wahl—UNRELATED: Consultancy: Agios Pharmaceuticals Inc, Comments: consulting fees for work related to radiation and brain tumors; Grants/Grants Pending: Agios Inc, Innocrin Inc, Comments: research funding for unrelated work regarding radiation resistance in brain tumors; Patents (Planned, Pending or (ssued): University of Michigan, Comments: patent pending related to methods to predict treatment responsiveness in brain tumors. Aditya S. Pandey-UNRELATED: Grants/Grants Pending: National Institutes of Healthbased grants for ultrasound-based treatment of intracerebral hemorrhage*; Patents (Planned, Pending Or Issued): ultrasound-based treatment of intracerebral hemorrhage, hypothermia tool, thrombectomy-based tools, Smart Drill, Comments: None of these have been issued, and none have been licensed or led to financial gain.* *Money paid to the institution.

\section{REFERENCES}

1. Lunsford LD, Kondziolka D, Flickinger JC, et al. Stereotactic radiosurgery for arteriovenous malformations of the brain. J Neurosurg 1991;75:512-24 CrossRef Medline

2. Steiner L, Lindquist C, Adler JR, et al. Clinical outcome of radiosurgery for cerebral arteriovenous malformations. J Neurosurg 1992;77:1-8 CrossRef Medline

3. Friedman WA, Bova FJ, Mendenhall WM. Linear accelerator radiosurgery for arteriovenous malformations: the relationship of size to outcome. J Neurosurg 1995;82:180-89 CrossRef Medline

4. Karlsson B, Lax I, Soderman M. Risk for hemorrhage during the 2year latency period following gamma knife radiosurgery for arteriovenous malformations. Int J Radiat Oncol Biol Phys 2001;49:104551 CrossRef Medline

5. Yen CP, Matsumoto JA, Wintermark M, et al. Radiation-induced imaging changes following gamma knife surgery for cerebral arteriovenous malformations. J Neurosurg 2013;118:63-73 CrossRef Medline

6. Cohen-Inbar $\mathrm{O}$, Lee $\mathrm{CC}, \mathrm{Xu} \mathrm{Z}$, et al. A quantitative analysis of adverse radiation effects following gamma knife radiosurgery for arteriovenous malformations. J Neurosurg 2015;123:945-53 CrossRef Medline

7. Hanauer DA, Mei Q, Law J, et al. Supporting information retrieval from electronic health records: a report of University of Michigan's nine-year experience in developing and using the Electronic Medical Record Search Engine (EMERSE). J Biomed Inform 2015;55:290-300 CrossRef Medline

8. Kothari RU, Brott T, Broderick JP, et al. The ABCs of measuring intracerebral hemorrhage volumes. Stroke 1996;27:1304-15 CrossRef Medline

9. Ganz JC, Reda WA, Abdelkarim K. Adverse radiation effects after gamma knife surgery in relation to dose and volume. Acta Neurochir (Wien) 2009;151:9-19 CrossRef Medline

10. Ilyas A, Chen CJ, Ding D, et al. Radiation-induced changes after stereotactic radiosurgery for brain arteriovenous malformations: a systematic review and meta-analysis. Neurosurgery 2018;83:365-76 CrossRef Medline

11. Kano H, Flickinger JC, Tonetti D, et al. Estimating the risks of adverse radiation effects after gamma knife radiosurgery for arteriovenous malformations. Stroke 2017;48:84-90 CrossRef Medline

12. Blamek $S$, Boba $M$, Larysz $D$, et al. The incidence of imaging abnormalities after stereotactic radiosurgery for cerebral arteriovenous and cavernous malformations. Acta Neurochir Suppl 2010;106:18790 CrossRef Medline

13. Vrdoljak E, Bill CA, Stephens LC, et al. Radiation-induced apoptosis of oligodendrocytes in vitro. Int J Radiat Biol 1992;62:475-80 CrossRef Medline

14. Hong JH, Chiang CS, Campbell IL, et al. Induction of acute phase gene expression by brain irradiation. Int J Radiat Oncol Biol Phys 1995;33:619-26 CrossRef Medline

15. Pollock BE. Occlusive hyperemia: a radiosurgical phenomenon? Neurosurgery 2000;47:1178-82; discussion 1182-74 CrossRef Medline

16. Chapman PH, Ogilvy CS, Loeffler JS. The relationship between occlusive hyperemia and complications associated with the radiosurgical treatment of arteriovenous malformations: report of two cases. Neurosurgery 2004;55:228-33; discussion 233-24 CrossRef Medline

17. van den Berg R, Buis DR, Lagerwaard FJ, et al. Extensive white matter changes after stereotactic radiosurgery for brain arteriovenous malformations: a prognostic sign for obliteration? Neurosurgery 2008;63:1064-69; discussion 1069-70 CrossRef Medline

18. Pollock BE, Link MJ, Branda ME, et al. Incidence and management of late adverse radiation effects after arteriovenous malformation radiosurgery. Neurosurgery 2017;81:928-34 CrossRef Medline

19. Williamson R, Kondziolka D, Kanaan H, et al. Adverse radiation effects after radiosurgery may benefit from oral vitamin $\mathrm{E}$ and pentoxifylline therapy: a pilot study. Stereotact Funct Neurosurg 2008;86:359-66 CrossRef Medline

20. Levin VA, Bidaut L, Hou P, et al. Randomized double-blind placebocontrolled trial of bevacizumab therapy for radiation necrosis of the central nervous system. Int J Radiat Oncol Biol Phys 2011;79:1487-95 CrossRef Medline 